\title{
Gene-expression molecular subtyping of triple-negative breast cancer tumours: importance of immune response
}

Pascal Jézéquel ${ }^{1,2,3,4^{*}}$, Delphine Loussouarn ${ }^{4,5}$, Catherine Guérin-Charbonnel 1,2,4, Loïc Campion ${ }^{4,6}$, Antoine Vanier ${ }^{7,8}$, Wilfried Gouraud ${ }^{1,2,4}$, Hamza Lasla ${ }^{2}$, Catherine Guette ${ }^{9}$, Isabelle Valo ${ }^{10}$, Véronique Verrièle ${ }^{10}$ and Mario Campone $e^{2,4,11}$

\begin{abstract}
Introduction: Triple-negative breast cancers need to be refined in order to identify therapeutic subgroups of patients.

Methods: We conducted an unsupervised analysis of microarray gene-expression profiles of 107 triple-negative breast cancer patients and undertook robust functional annotation of the molecular entities found by means of numerous approaches including immunohistochemistry and gene-expression signatures. A triple-negative external cohort $(n=87)$ was used for validation.

Results: Fuzzy clustering separated triple-negative tumours into three clusters: C1 (22.4\%), C2 (44.9\%) and C3 (32.7\%). $C 1$ patients were older (mean $=64.6$ years) than $C 2$ (mean $=56.8$ years; $P=0.03$ ) and C3 patients (mean $=51.9$ years; $P=0.0004)$. Histological grade and Nottingham prognostic index were higher in $C 2$ and $C 3$ than in $C 1(P<0.0001$ for both comparisons). Significant event-free survival $(P=0.03)$ was found according to cluster membership: patients belonging to $C 3$ had a better outcome than patients in $C 1(P=0.01)$ and $C 2(P=0.02)$. Event-free survival analysis results were confirmed when our cohort was pooled with the external cohort $(n=194 ; P=0.01)$. Functional annotation showed that $22 \%$ of triple-negative patients were not basal-like (C1). C1 was enriched in luminal subtypes and positive androgen receptor (luminal androgen receptor). C2 could be considered as an almost pure basal-like cluster. C3, enriched in basal-like subtypes but to a lesser extent, included $26 \%$ of claudin-low subtypes. Dissection of immune response showed that high immune response and low M2-like macrophages were a hallmark of C3, and that these patients had a better event-free survival than C2 patients, characterized by low immune response and high M2-like macrophages: $P=0.02$ for our cohort, and $P=0.03$ for pooled cohorts.

Conclusions: We identified three subtypes of triple-negative patients: luminal androgen receptor (22\%), basal-like with low immune response and high M2-like macrophages (45\%), and basal-enriched with high immune response and low M2-like macrophages (33\%). We noted out that macrophages and other immune effectors offer a variety of therapeutic targets in breast cancer, and particularly in triple-negative basal-like tumours. Furthermore, we showed that CK5 antibody was better suited than CK5/6 antibody to subtype triple-negative patients.
\end{abstract}

\footnotetext{
* Correspondence: Pascal.jezequel@ico.unicancer.fr

'Unité Mixte de Génomique du Cancer, Institut de Cancérologie de l'Ouest

site René Gauducheau, Bd J. Monod, 44805 Saint Herblain Cedex, France

${ }^{2}$ Unité de Bioinfomique, Institut de Cancérologie de l'Ouest - site René

Gauducheau, Bd J. Monod, 44805 Saint Herblain Cedex, France

Full list of author information is available at the end of the article
} 


\section{Introduction}

Breast cancer heterogeneity makes it difficult to bring personalized medicine into the clinic. For many years, research has been aimed at deciphering molecular presentation of this disease to identify subgroups of patients with clinical significance, such as prognosis or response to therapy, in order to optimize patient management. Immunohistochemistry (IHC)-typed triple-negative (TN) tumours, which represent 12 to $17 \%$ of primary breast cancer, are among the most aggressive and deadly breast cancer subtypes [1]. The lack of oestrogen, progesterone and HER2 receptors makes its therapeutic management optimisation difficult. Furthermore, TN breast cancer tumours are also known to be heterogeneous. The era of large-scale science, which is linked both to recent technological advances and to the availability of full genetic information, has boosted the research for new biomarkers and molecular subtyping. In 2000 and 2001, breast tumour gene-expression profiling (GEP) revealed five intrinsic subtypes: luminal A, luminal B, HER2enriched (HER2-E), basal-like and normal breast-like $[2,3]$. Luminal A tumours were associated with a better prognosis in comparison with other subtypes, which displayed similar bad prognoses. Luminal and HER2-E subtypes defined subgroups of patients who could receive targeted therapies (hormonotherapy and trastuzumab, respectively). Basal-like tumours are often mixed up with TN tumours; the main difference comes from the typing method (GEP for basal-like and IHC for TN). Comparative studies showed that not all TN tumours are identified as basal-like (approximately 80\%), and not all basal-like tumours are TN $[1,4]$. Both types affect younger patients, and are linked to a bad prognosis and no possibility of targeted therapy. Other works refined TN subtyping and identified a new molecular entity, named claudin-low, characterized by low expression of cell-cell adhesion cluster containing claudin 3, 4, 7 and E-cadherin, luminal and proliferation-associated genes, enrichment in epithelial-tomesenchymal transition (EMT) features, immune system responses, and stem cell-associated biological processes $[5,6]$. No prognostic difference was noted between basallike and claudin-low patients [6]. An interferon-rich subgroup, characterized by high expression of interferonregulated genes, was further discovered [7]. Gene Ontology (GO) categories of these genes underlined "immune response" and "defense response". Later, six molecular subtypes were also identified in TN: basal-like 1, basallike 2, immunomodulatory, mesenchymal-like, mesenchymal stem-like, and luminal androgen receptor (LAR) [8]. Today, heterogeneity within basal-like and TN is still controversial and requires further research, including new cohorts, to understand the complexity of the disease, and to identify molecular drivers that could be therapeutically targeted $[1,6]$.
In this study, we conducted an unsupervised analysis of a new cohort of $107 \mathrm{TN}$ breast cancer patients and undertook robust functional annotation of the main molecular entities found by means of numerous complementary approaches.

\section{Methods}

\section{Patients}

The bi-centric studied cohort retrospectively included 107 randomly selected women whose primary breast tumours lacked immunohistochemical expression of oestrogen receptor (ER), progesterone receptor (PR) and HER2; hence subtyped as TN tumours. Patients were diagnosed and treated primarily between 1998 and 2007 at the Institut de Cancérologie de l'Ouest - René Gauducheau $(n=65)$ and the Institut de Cancérologie de l'Ouest - Paul Papin $(n=42)$. Of the 107 patients, 44 relapsed and 63 were disease-free after the follow-up period (median followup $=7$ years). All patients showed no evidence of relapse at the time of diagnosis. None had received chemotherapy, endocrine therapy or radiation therapy prior to surgery. Treatment decisions and follow-up processes were based solely on international recommendations. The follow-up data of patients included clinical examination and mammography every 6 months for 2 years and annually thereafter. Informed consent was obtained from patients to use their surgical specimens and clinicopathological data for research purposes, as required by the French Committee for the Protection of Human Subjects (CCPPRB). Ouest IV - Nantes CCPPRB approved use of tumour tissues for this study (6 May 2013: $\mathrm{n}^{\circ}$. 357/2013). Collection of tumours was approved by French Minister of higher education and research $\left(n^{\circ}\right.$. AC-2008-141). This study did not need additional ethical approval.

\section{Tumour tissues}

All tumour tissue samples were surgically collected and processed in two parts by a pathologist. The first part was fixed in $10 \%$ neutral buffered formalin for standard histological analysis and IHC. The second part was immediately macrodissected, snap-frozen in liquid nitrogen and stored until RNA extraction.

\section{RNA extraction}

Total RNA was prepared following standard protocols then treated with Dnase I using the RNeasy column purification system (Qiagen, Courtaboeuf, France). Assessment of RNA quality, integrity and purity was done through a Bionalyser 2100 (Agilent Technologies, Palo Alto, CA, USA). RNA samples were considered for further analysis only if they had distinct $28 \mathrm{~S}$ and $18 \mathrm{~S}$ ribosomal peaks. 


\section{Gene expression profiling}

Gene expression analysis was performed using Affymetrix ${ }^{\circ}$ Human Genome U133 Plus 2.0 Arrays (Affymetrix ${ }^{\circ}$, Santa Clara, CA, USA) measuring over 43,000 transcripts representing over 20,000 human genes. cRNA synthesis and labelling, as well as chip hybridisation, washing and image scanning were performed according to the manufacturer's protocol. All microarrays complied with quality criteria. Microarray and patient clinical data have been deposited in the Gene Expression Omnibus (GEO) under the GSE58812 accession number.

\section{Bioinformatics}

\section{Internal and external data pre-processing}

Bioclinical and expression data of the external cohort used for validation were available from the GEO, accession number GSE21653 [9,10]. For both our cohort and the external cohort, the Affymetrix ${ }^{\circ}$ CEL files (raw data) were MAS5-normalized in the Affymetrix Expression Console (v1.3.1) and then log2-transformed.

\section{Unsupervised analysis}

To organise data into groups with the same underlying molecular characteristics, we performed clustering analysis based on the $5 \%$ most variable probe sets $(n=2,734)$ by means of the fuzzy clustering method. Fuzzy clustering permits each patient to have a probability of membership to each cluster along with the cluster number, with the sum of all cluster membership probabilities being 1 for any patient. Briefly, the method is based on the minimization of $\sum_{k=1}^{n} \frac{\sum_{i, j} u(i, k)^{r} u(j, k)^{r} d(i, j)}{2 \sum_{j} u(j, k)^{r}}$, where $n$ is the number of clusters, $\mathrm{i}$ and $\mathrm{j}$ represent the patients, $\mathrm{u}(\mathrm{i}, \mathrm{k})$ is the membership of patient $i$ for cluster $k, r$ is the fuzziness index and $d(i, j)$ is the dissimilarity between patients $i$ and $j$ (centred Pearson distance in our study). To decide how many clusters were present in our data we calculated two indices, the Dunn index and the Calinski and Harabasz index, considering two to 10 clusters, but we also performed a hierarchical clustering to visualise the partition and study the concordance of both clustering methods. All computations showed either two or three clusters (similar results for both numbers). It was decided to create three clusters and study the membership probabilities.

\section{Cluster functional annotation}

To annotate the clusters found, we used clinicopathologic characteristics, 10 IHC markers, 17 gene-expression signatures (GES), GO biological process terms enrichment, and intuitive single gene-expression approach. Differences among the three clusters for the different characteristics were assessed by analysis of variance (along with Tukey's
HSD (honest significant differences) test in case of significance) for continuous variables, Fisher's exact test for categorical variables, and Cox regression model or KaplanMeier survival curves (along with logrank test) for survival data. Correlations between GES were measured by Pearson's correlation coefficient.

\section{Tissue microarrays and imunohistochemistry Tissue microarray construction}

Hemalun-eosin-safran (HES)-stained sections were reviewed and the area of interest was marked out on the slide. For each case, three representative tumour areas were selected from the HES-stained slide of the paraffinembedded donor block. Using a tissue arrayer (Beecher Instruments, Sun Prairie, WI, USA), 1-mm diameter cores of breast cancer tissue were punched out from the paraffin-embedded tissue block and placed into recipient paraffin blocks. A control tissue sample (normal breast) was included in each recipient paraffin block. Before immunostaining, HES-stained slides of the final array blocks were examined to confirm the representative areas of the tumours in comparison to the original HES-stained section.

\section{Immunohistochemistry and scoring}

Sections of paraffin-embedded tissue microarray blocks ( $4 \mu \mathrm{m}$ thick) were deparaffinized in xylene and rehydrated through a graded series of alcohol. IHC was performed using the EnVision Detection Systems (Dako, Les Ulis, France) according to the manufacturer's instructions; 3,3'-diaminobenzidine was used as a chromogen. The sections were counterstained with haematoxylin. Details of the antigen retrieval technique and dilution of the primary antibodies (CK5/6, CK5, HER1, androgen receptor (AR) Ki-67, FOXA1, E-cadherin, claudin 3, claudin 4 and claudin 7) are described in Additional file 1. Immunostaining results were assessed by a pathologist in a blinded manner. Immunoreactivity of each target in the tissue microarray cores was scored as a percentage of cells stained and/or staining intensity. Immunohistochemical staining interpretation is displayed in Additional file 2.

\section{Gene-expression signatures}

Seventeen GES were used to annotate the three clusters: three single sample predictors (SSPs) (Sorlie's SSP, Hu's SSP and Parker's SSP (PAM50)), proliferation score, a subtyping tool for TN breast cancer (TNBCtype), Teschendorff's GES, vascular endothelial growth factor (VEGF) profile, glycolysis profile, claudin-low signature and seven immune metagenes [6-8,11-16]. Furthermore, in order to discriminate macrophages into M1-like or M2-like subpopulations, which could not be identified by Rody's HCK metagene, we established an M2/M1 GES based on Beyer's microarray data (GSE35449) [17]. The significance 
analysis of microarrays (SAM) method was applied to find the probes differentially expressed between M1 and M2 populations, and genes for which all corresponding probes exhibited a $Q$-value of $0 \%$ were retained. The GES was then computed in our cohort as the weighted mean of the genes retained, weights being +1 or -1 , depending on the expression of each gene in M2 relative to M1. When a gene was represented by multiple probe sets, the median of the different probe sets was taken as the unique value for the gene. Methods are summarized in Additional file 3.

\section{Single gene-expression intuitive approach}

An intuitive approach was also used to annotate patient clusters. Based on breast cancer transcriptomic studies, we selected representative genes $(n=49)$ of molecular subtypes (luminal, HER2-E, basal-like, claudin-low) and biological processes (proliferation, breast stem cells, EMT, cell migration, immune system response and angiogenesis) $[6,18]$. Their expressions were compared in function of clusters.

\section{Gene ontology biological process terms enrichment}

Functional annotation of each cluster through biological process analysis was performed using DAVID bioinformatics resources 6.7 (the Database for Annotation, Visualization, and Integrated Discovery), Gorilla and ToppGene web tools to discover the GO categories with significantly enriched gene numbers [19-22]. Two methods were used to select genes differentially expressed across the clusters. The SAM method was performed to obtain lists of genes with significantly different expression between clusters (one versus the others and two-by-two); genes for which all corresponding probe sets had a $Q$ value of $0 \%$ were retained. In addition to the SAM method, expression of the 2,734 most variable probe sets was represented on a heatmap, with patients ordered according to the fuzzy clusters. Ward hierarchical clustering was performed on the probe sets, and clusters of corresponding genes with visually differential expression among the clusters of patients were retained.

\section{Unsupervised external validation process}

The same global process as the one applied to our cohort was also applied to the TN patients of the external cohort (GSE21653) to validate unsupervised analysis results. Moreover, the prediction analysis for microarrays (PAM) method was used to predict clusters obtained with our cohort - the predictor was trained on our cohort and then applied to GSE21653 TN patients; the predict clusters were then compared to the ones obtained by fuzzy clustering.

\section{Statistical analysis}

All statistical analyses were performed with $\mathrm{R}$ [23] and the packages amap, cluster, ggplots, grid, pamr, samr, survival. All $P$-values are two-sided; $P$-values less than $5 \%$ are considered significant.

\section{Results}

\section{External data selection and pre-processing}

We selected the GSE21653 cohort for external validation because the status of ER, PR and HER2 were available and gene expressions were measured with the same DNAchip as for our cohort. Of the 266 patients that composed the GSE21653 cohort, 87 patients were TN.

\section{Unsupervised analysis}

We chose a fuzzy clustering method to investigate molecular differences among our TN cohort because it keeps the possibility for each patient to belong to multiple clusters at the same time but with different "degrees of membership". Fuzzy clustering separated TN tumours into three clusters, named $\mathrm{C} 1(n=24 ; 22.4 \%)$, C2 $(n=48 ; 44.9 \%)$ and C3 $(n=35 ; 32.7 \%)$ (Figure 1$)$. Distribution of samples in all three clusters was independent of a patient's origin $(n=2)(P=0.25)$. Fuzzy clustering probabilities demonstrated that $\mathrm{C} 1$ was more robust than $\mathrm{C} 2$ and $\mathrm{C} 3(\mathrm{C} 1$ : minimum of probabilities = $73 \%$, median $=98 \%, 20 \mathrm{C} 1$ patients $(83 \%)$ with probability $>90 \%$; C2: minimum of probabilities $=49 \%$, median $=$ 79\%, 36 C2 patients (75\%) with probability <90\%; C3: minimum of probabilities $=42 \%$, median $=85 \%, 19 \mathrm{C} 3$ patients (54\%) with probability <90\%) and that $\mathrm{C} 2$ and C3 were very close. If we consider only the C2 and C3 patients, the smallest difference between the probability

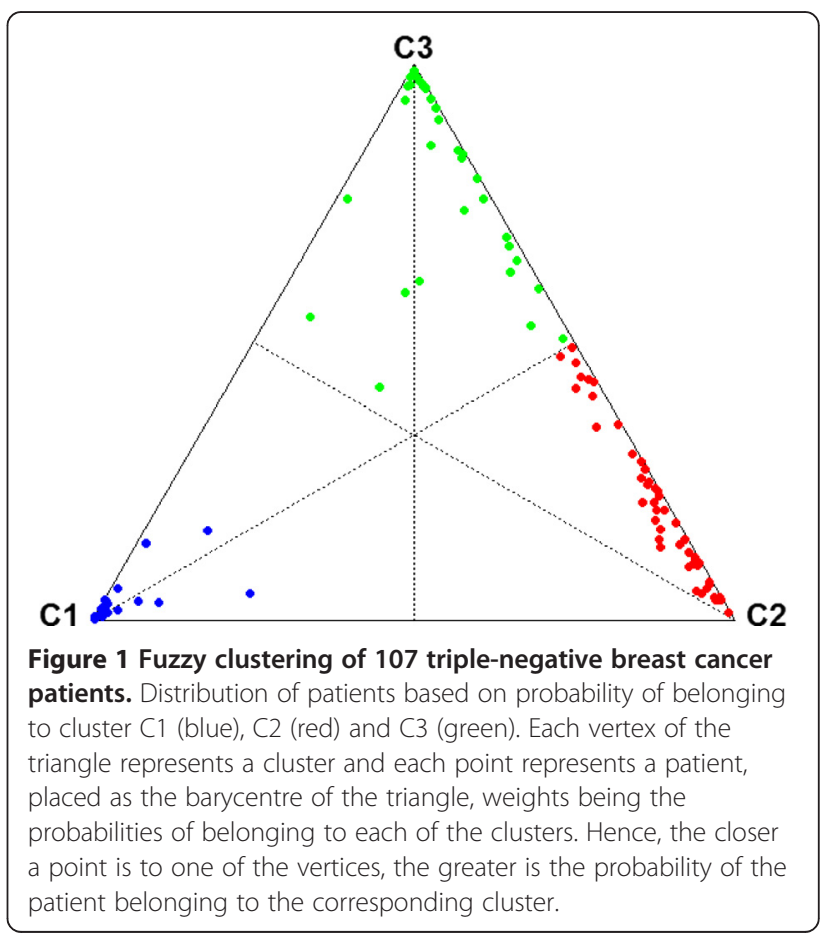


of belonging to $\mathrm{C} 2$ and the one of belonging to $\mathrm{C} 3$ was only $1 \%$; for 11 patients (13\%), this difference was below $20 \%$.

\section{Cluster functional annotation}

Cluster functional annotation results are detailed in the five following sections: clinicopathologic characteristics, IHC, GES, single gene-expression intuitive approach and GO biological process terms enrichment.

\section{Clinicopathologic characteristics}

We have selected 107 breast carcinomas that were shown to lack IHC expression of ER, PR and HER2. The clinicopathologic characteristics of these 107 tumours are displayed in Table 1 . Patients belonging to $\mathrm{C} 1$ were older (mean $=64.6$ years) than patients included in $\mathrm{C} 2$ (mean $=56.8$ years; $P=0.03$ ) and in $\mathrm{C} 3$ (mean $=51.9$ years; $P=0.0004)$. No significant age difference was observed between $\mathrm{C} 2$ and $\mathrm{C} 3(P=0.17)$. Histological grade and Nottingham prognostic index (NPI) were higher in $\mathrm{C} 2$ and $\mathrm{C} 3$ than in $\mathrm{C} 1$. These results showed that $\mathrm{C} 1$ was different from $\mathrm{C} 2$ and $\mathrm{C} 3$, and that $\mathrm{C} 1$ seemed to be characterized by a less aggressive illness according to age, histological grade and NPI. A significant event-free survival
(EFS) $(P=0.0321)$ and a trend for overall survival (OS) $(P=0.0653)$ were found according to cluster membership (Figure 2). Patients belonging to $\mathrm{C} 3 \mathrm{had}$ a better EFS than patients in $\mathrm{C} 1(P=0.0145)$ and $\mathrm{C} 2(P=0.0195)$. No outcome difference existed between $\mathrm{C} 1$ and $\mathrm{C} 2(P=0.76)$. EFS results were confirmed when our cohort was pooled with the GSE21653 TN patients $(P=0.01)$ (Additional file 4). Patients belonging to $C 3$ had a better EFS than patients in $\mathrm{C} 1(P=0.03)$ and $\mathrm{C} 2(P=0.002)$, and no outcome difference was observed between $\mathrm{C} 1$ and $\mathrm{C} 2(P=$ 0.45). One main reason might explain why patients belonging to $\mathrm{C} 1 \mathrm{had}$ a poor prognosis; $\mathrm{C} 1$ included numerous PAM50 luminal subtypes (10 out of 24 ; mainly luminal B (A, $n=1 ; \mathrm{B}, n=9)$ ), which are characterized by a poor outcome. Furthermore, these luminal patients did not receive hormonotherapy. The same observation was made for GSE21653 and the equivalent cluster C1, which included several luminal patients (7/27 (A, $n=1$; $\mathrm{B}, n=6)$ ).

\section{Immunohistochemistry}

Basal-like phenotype, as defined by Nielsen and colleagues [24] (ER negative and HER2 negative, and CK5/

Table 1 Clinicopathologic characteristics of the studies triple-negative tumours

\begin{tabular}{|c|c|c|c|c|c|}
\hline Variable & $\begin{array}{l}\text { All } \\
(n=107)\end{array}$ & $\begin{array}{l}\text { Cluster } 1 \\
(n=24)\end{array}$ & $\begin{array}{l}\text { Cluster } 2 \\
(n=48)\end{array}$ & $\begin{array}{l}\text { Cluster } 3 \\
(n=35)\end{array}$ & $P$-value \\
\hline Age (years; mean $\pm \mathrm{SD}$ ) & $56.9 \pm 12.8$ & $64.6 \pm 10.6$ & $56.8 \pm 12.9$ & $51.9 \pm 11.7$ & 0.0006 \\
\hline \multicolumn{6}{|l|}{ SBR grade } \\
\hline 1 & 2 & 2 & 0 & 0 & \\
\hline 2 & 14 & 10 & 1 & 3 & \\
\hline 3 & 91 & 12 & 47 & 32 & $<0.0001$ \\
\hline Tumour size $(\mathrm{mm}$; mean $\pm \mathrm{SD})$ & $22.3 \pm 12.7$ & $27.3 \pm 18.3$ & $22.8 \pm 12.1$ & $18.5 \pm 7.0$ & 0.184 \\
\hline \multicolumn{6}{|l|}{ Nodal status } \\
\hline 0 & 77 & 17 & 38 & 22 & \\
\hline 1 & 30 & 7 & 10 & 13 & 0.266 \\
\hline \multicolumn{6}{|l|}{ NPI } \\
\hline 1 & 13 & 9 & 1 & 3 & \\
\hline 2 & 71 & 8 & 40 & 23 & \\
\hline 3 & 22 & 6 & 7 & 9 & $<0.0001$ \\
\hline \multicolumn{6}{|l|}{ Radiotherapy } \\
\hline No & 4 & 2 & 1 & 1 & \\
\hline Yes & 103 & 22 & 47 & 34 & 0.436 \\
\hline \multicolumn{6}{|l|}{ Adjuvant therapy } \\
\hline No & 12 & 6 & 4 & 2 & \\
\hline Yes & 95 & 18 & 44 & 33 & 0.071 \\
\hline \multicolumn{6}{|l|}{ Hormonotherapy } \\
\hline No & 106 & 23 & 48 & 35 & \\
\hline Yes & 1 & 1 & 0 & 0 & 0.224 \\
\hline
\end{tabular}

NPI, Nottingham prognostic index; SBR, Scarff Bloom Richardson. 

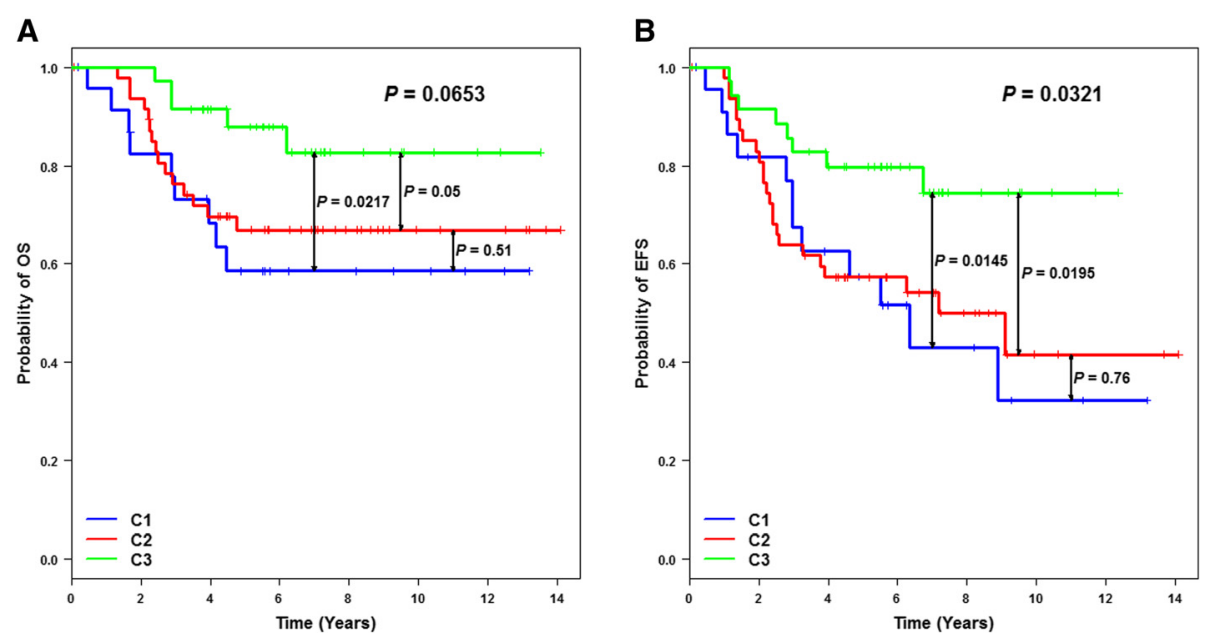

Figure 2 Kaplan-Meier analyses of 107 triple-negative breast cancer patients based on fuzzy-clustering partition. (A) Overall survival (OS) analysis shows that $C 3$ patients have a better outcome than $C 1(P=0.0217)$ and $C 2$ patients $(P=0.05)$. (B) Event-free survival (EFS) analysis shows the same result: $C 3 / C 1, P=0.0145 ; C 3 / C 2, P=0.0195$.

6 and/or HER1 positive) was found in $50 \%$ of $\mathrm{C} 1 \mathrm{pa}$ tients (the same result was found with CK5), and $71 \%$ or $92 \%$ in $\mathrm{C} 2+\mathrm{C} 3$ patients by means of CK5/6 or CK5, respectively. Our results showed that CK5 IHC expression was more concordant with gene expression profiling results than CK5/6: $92 \%$ of $\mathrm{C} 2+\mathrm{C} 3$ patients were typed as basal-like by means of CK5 and/or HER1 positivity and $71 \%$ by means of CK5/6 and/or HER1 positivity. Furthermore, considering CK 5 and CK5/6 alone, $83 \%$ of $\mathrm{C} 2+\mathrm{C} 3$ patients showed positivity for CK5 but only $34 \%$ had positivity for CK5/6 $(P=0.0019)$. Hence, CK5 should be used to demonstrate basal-like characteristics, as underlined by a few studies $[25,26]$. Briefly, functional annotation of fuzzy cluster results by means of IHC markers showed that $\mathrm{C} 1$ was not a basal-like cluster (AR and FOXA1 positive, low Ki-67 expression) and, on the contrary, that C2 and $\mathrm{C} 3$ were basal-like (CK5 and/or HER1 positive, high Ki-67 expression) (Table 2). C2 and C3 could not be distinguished based on IHC claudin-low markers (claudin 3, 4,7 and E-cadherin). No statistical link was found between low claudin 3, 4 or 7 and claudin-low subtype as defined by Prat $(P=0.99, P=0.56, P=0.35$, respectively) or between IHC claudin-low (claudin 3, 4, 7 negative and/ or E-cadherin negative) and claudin-low $(P=0.52)$ [6].

\section{Gene-expression signatures}

GES subtyping results are discussed below and detailed in Table 3 and Figure 3.

Single sample predictor annotation Assignment of patients to a particular molecular subtype by means of SSP is dependent on the SSP used; for this reason, we used three available SSPs [27]. These signatures showed that C1 essentially contained non-basal-like subtypes (Table 3,
Additional file 5). This cluster was mostly composed of luminal $A$ and $B$ subtypes, and unclassified tumours. In C1, PAM50 subtyping identified $79.1 \%$ of luminal subtypes (luminal A (20.8\%) and B (58.3\%)) and only one basal-like tumour. According to luminal enrichment of $\mathrm{C} 1$ and IHC results for AR, C1 should be named LAR. $\mathrm{C} 2$ was an almost pure basal-like cluster whatever the SSP used. In C2, one patient, who was subtyped as normal breast-like by means of PAM50, unclassified by Hu's SSP and basal-like by Sorlie's SSP, presented an adenoid cystic carcinoma, which is considered as a TN phenotype

Table 2 Functional annotation of fuzzy clusters by means of immunohistochemistry

\begin{tabular}{|c|c|c|c|c|}
\hline Marker interpretation & $\begin{array}{l}\text { C1 } \\
(n=24)\end{array}$ & $\begin{array}{l}\text { C2 } \\
(n=48\end{array}$ & $\begin{array}{l}\text { C3 } \\
(n=35)\end{array}$ & $P$-value \\
\hline CK5/6 and/or HER1 positive & $50 \%$ & \multicolumn{2}{|c|}{$71 \%$} & 0.077 \\
\hline CK5 and/or HER1 positive & $50 \%$ & \multicolumn{2}{|c|}{$92 \%$} & $<10^{-5}$ \\
\hline Ki-67 positive & $29 \%$ & \multicolumn{2}{|c|}{$87 \%$} & $2.10^{-7}$ \\
\hline AR positive & $73 \%$ & \multicolumn{2}{|r|}{$5 \%$} & $<10^{-9}$ \\
\hline FOXA1 positive & $73 \%$ & \multicolumn{2}{|c|}{$5 \%$} & $7.10^{-13}$ \\
\hline \multirow[t]{2}{*}{ E-cadherin positive } & $64 \%$ & \multicolumn{2}{|c|}{$48 \%$} & 0.197 \\
\hline & - & $42 \%$ & $56 \%$ & 0.236 \\
\hline \multirow[t]{2}{*}{ Claudin 3 positive } & $38 \%$ & \multicolumn{2}{|c|}{$59 \%$} & 0.085 \\
\hline & - & $58 \%$ & $60 \%$ & 0.879 \\
\hline \multirow[t]{2}{*}{ Claudin 4 positive } & $91 \%$ & \multicolumn{2}{|c|}{$93 \%$} & 0.672 \\
\hline & - & $92 \%$ & $94 \%$ & 0.990 \\
\hline \multirow[t]{2}{*}{ Claudin 7 positive } & $18 \%$ & \multicolumn{2}{|c|}{$12 \%$} & 0.485 \\
\hline & - & $12 \%$ & $11 \%$ & 0.990 \\
\hline \multirow{2}{*}{$\begin{array}{l}\text { Claudin 3, 4, } 7 \text { negative and/or } \\
\text { E-cadherin negative }\end{array}$} & $38 \%$ & \multicolumn{2}{|c|}{$52 \%$} & 0.261 \\
\hline & - & $58 \%$ & $43 \%$ & 0.163 \\
\hline
\end{tabular}


Table 3 Gene-expression profiling of the three clusters

\begin{tabular}{|c|c|c|c|c|c|}
\hline GES name & & $\begin{array}{l}\text { Cluster } 1 \\
(n=24)\end{array}$ & \multirow{2}{*}{$\begin{array}{l}\text { Cluster } 2 \\
(n=48)\end{array}$} & $\begin{array}{l}\text { Cluster } 3 \\
(n=35)\end{array}$ & $P$-value \\
\hline \multirow{6}{*}{ Sorlie's SSP } & & & & & \\
\hline & Basal-like & 0 & 48 & 23 & $<0.0001$ \\
\hline & HER2-E & 1 & 0 & 0 & \\
\hline & Luminal A & 1 & 0 & 0 & \\
\hline & Luminal B & 9 & 0 & 8 & \\
\hline & Unclassified & 13 & 0 & 4 & \\
\hline \multirow[t]{5}{*}{ Hu's SSP } & Basal-like & 0 & 47 & 30 & $<0.0001$ \\
\hline & HER2-E & 0 & 0 & 0 & \\
\hline & Luminal A & 8 & 0 & 0 & \\
\hline & Luminal B & 0 & 0 & 0 & \\
\hline & Unclassified & 16 & 1 & 5 & \\
\hline \multirow[t]{6}{*}{ Parker's SSP = PAM50 } & Basal-like & 1 & 47 & 32 & $<0.0001$ \\
\hline & HER2-E & 0 & 0 & 0 & \\
\hline & Luminal A & 5 & 0 & 0 & \\
\hline & Luminal B & 14 & 0 & 2 & \\
\hline & NBL & 3 & 1 & 1 & \\
\hline & Unclassified & 1 & 0 & 0 & \\
\hline Proliferation score (mean \pm sd) & & $8.64 \pm 0.97$ & $10.22 \pm 0.63$ & $9.74 \pm 0.57$ & $<0.0001$ \\
\hline \multirow[t]{7}{*}{ TNBCtype } & BL1 & 0 & 20 & 5 & $<0.0001$ \\
\hline & BL2 & 2 & 3 & 1 & \\
\hline & $\mathrm{IM}$ & 0 & 0 & 19 & \\
\hline & LAR & 8 & 0 & 0 & \\
\hline & M & 0 & 18 & 0 & \\
\hline & MSL & 3 & 1 & 4 & \\
\hline & Unclassified & 11 & 6 & 6 & \\
\hline \multirow[t]{5}{*}{ Teschendorff's GES } & $\mathrm{CC}+$ & 0 & 7 & 0 & \\
\hline & $\mathrm{CC}+/ \mathrm{IR}+$ & 0 & 41 & 33 & \\
\hline & IR+ & 1 & 0 & 1 & \\
\hline & SR+ & 13 & 0 & 0 & \\
\hline & Unclassified & 10 & 0 & 1 & \\
\hline VEGF profile (mean \pm SD) & & $9.20 \pm 0.49$ & $9.85 \pm 0.51$ & $9.53 \pm 0.42$ & $<0.0001$ \\
\hline Glycolysis profile (mean \pm SD) & & $10.48 \pm 0.55$ & $10.84 \pm 0.46$ & $10.63 \pm 0.43$ & 0.0073 \\
\hline \multirow[t]{2}{*}{ Claudin-low } & Claudin-low & 0 & 1 & 9 & 0.0002 \\
\hline & Other & 24 & 47 & 26 & \\
\hline
\end{tabular}

BL, basal-like; CC, cell cycle; GES, gene-expression signatures; IM, immunomodulatory; IR, immune response; LAR, luminal androgen receptor; $M$, mesenchymal; MSL, mesenchymal stem-like; NBL, normal breast-like; SR, steroid hormone receptor; SSP, single sample predictor; VEGF, vascular endothelial growth factor.

with an excellent prognosis [28]. This could explain why this tumour had a different molecular assignment compared to other C2 tumours. C3 included mostly basal-like subtypes, but to a lesser extent than C2 (91.4\% by PAM50 SSP, $85.7 \%$ by Hu's SSP and $65.7 \%$ by Sorlie's SSP).

Proliferation score Proliferation score significantly decreased from $\mathrm{C} 2$ to $\mathrm{C} 3(P<0.0074)$, then from $\mathrm{C} 3$ to $\mathrm{C} 1$ $(P<0.0001)$. It is important to notice that the two basal- like-enriched clusters ( $\mathrm{C} 2$ and $\mathrm{C} 3$ ) presented a significantly different proliferation score.

TNBCtype TNBCtype classification assigned a TNBC subtype to $78.5 \%$ of our tumours (Table 3, Additional file 6). Most of the unstable (that is, unclassified) tumours belonged to C1 (48\%). C1 was LAR-enriched (61.5\% of classified patients), and LAR subtypes were exclusively assigned to this cluster. This result confirmed that $\mathrm{C} 1$ was 


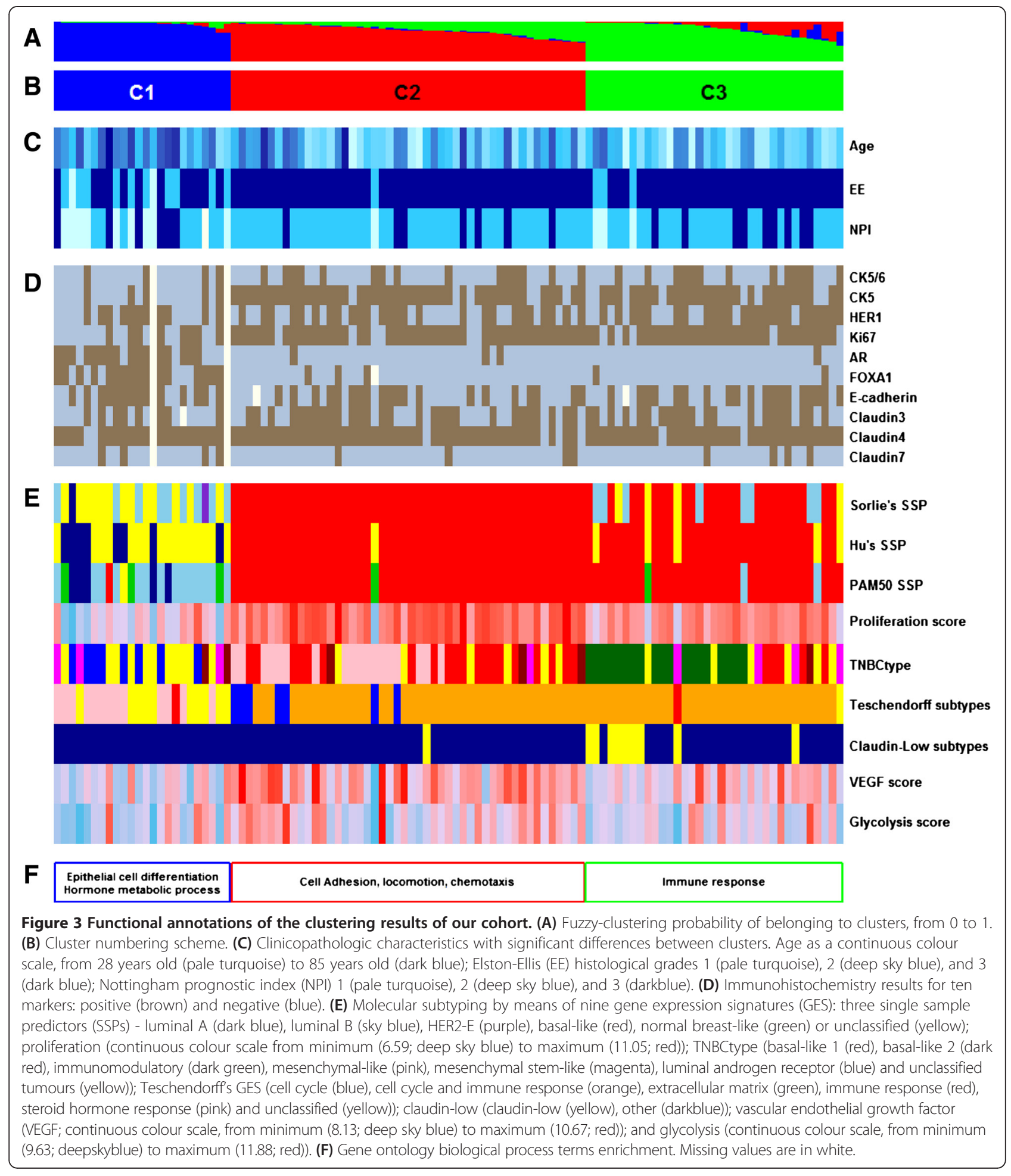

not a basal-like cluster as shown with the SSPs. C2 was basal-like 1 enriched and mesenchymal enriched (47.6\% and $42.8 \%$, respectively). Basal-like 1 and basal-like 2 represented $55 \%$ of $\mathrm{C} 2$. C3 was immunomodulatory enriched and mesenchymal stem-like enriched $(65.5 \%$ and $13.8 \%$, respectively). Mesenchymal stem-like, characterized by low expression of proliferation genes compared to mesenchymal-like, was mostly found in C1 and C3. All immunomodulatory subtypes were included in C3. Immune response distinguished $\mathrm{C} 3$ from $\mathrm{C} 2$. Distribution of TNBCtype subtypes as a function of PAM50 basal-like or non-basal-like led to the same conclusion as Masuda and 
colleagues [29] - non-PAM50 basal-like subtypes (C1 in our study) were mostly composed of LAR and mesenchymal stem-like subtypes (unclassified excluded).

Teschendorff's gene-expression signature Teschendorff's GES has been designed for ER-negative tumour subtyping. Steroid hormone receptor, cell cycle $(\mathrm{CC}+)$ and cell cycle and immune response $(\mathrm{CC}+/ \mathrm{IR}+)$ subtypes were exclusively observed in $\mathrm{C} 1, \mathrm{C} 2$ and $\mathrm{C} 2+\mathrm{C} 3$, respectively (Figure 3). Of note, most frequently immune response was mixed with cell cycle $(\mathrm{CC}+/ \mathrm{IR}+)(n=74)$. Only two "pure" IR+ subtypes were found (one in $\mathrm{C} 1$ and one in C3). This GES confirmed TNBCtype subtyping of $\mathrm{C} 1$ as steroid hormone receptor and LAR. Immune response was almost exclusively assigned to $\mathrm{C} 2$ and $\mathrm{C} 3$ but did not separate these two clusters.

Vascular endothelial growth factor profile This 13GES showed that angiogenesis varied according to the three clusters $(P<0.0001)$. Angiogenesis increased from C1 to C3 $(P=0.0274)$, and then from C3 to C2 $(P=$ $0.0114)$.

Glycolysis profile Glycolysis score was significantly different according to cluster $(P=0.0073)$. The score of $\mathrm{C} 1$ was inferior to that of $\mathrm{C} 2(P=0.0073)$ but no different from that of $\mathrm{C} 3(P=0.44)$. A slight trend was found between $\mathrm{C} 2$ and $\mathrm{C} 3(P=0.12)$.

Signature correlation analyses VEGF/glycolysis, proliferation/VEGF and proliferation/glycolysis profiles were positively correlated for patients included in our TN cohort $(\mathrm{r}=0.62, P<0.0001 ; \mathrm{r}=0.41, P<0.0001 ; \mathrm{r}=0.48, P<$ 0.0001 , respectively). Furthermore, correlation between VEGF and glycolysis profiles was found in each cluster (C1: $\mathrm{r}=0.63, P=0.0009 ; \mathrm{C} 2: \mathrm{r}=0.57, P<0.0001 ; \mathrm{C} 3: \mathrm{r}=$ $0.52, P=0.0015)$. It is important to note that the gene lists for VEGF score, glycolysis score and proliferation score have no gene in common and, thus, correlations can be attributed to tumour biology.

Claudin-low Ten patients were subtyped as "claudinlow" (9.3\%). The distribution was: $0 \%$ in $\mathrm{C} 1,2 \%(1 / 48)$ in C2 and 26\% (9/35) in C3. Thus, C3 was claudin-low enriched but this subtype only represented a quarter of its number. Claudin-low compared to PAM50 basal-like tumours $(n=70)$ showed significant lower proliferation $(P=0.0076$, Wilcoxon's test). This result is in concordance with current knowledge. Prat and colleagues concluded that these tumours were likely slower-cycling tumours [6].

Rody's gene expression signature In order to dissect immune response, we applied Rody's immune metagenes to patients belonging to $\mathrm{C} 2$ and $\mathrm{C} 3$. In doing so, seven immune modules representing immune cells or immune processes were distinguished: B lymphocytes (IgG); macrophages and monocyte/myeloid lineage cells (HCK); professional antigen-presenting cells (MHC-II); $\mathrm{T}$ cells (LCK); cell types for presentation of intracellular antigens (MHC-I); interferon signal transduction (STAT1); and interferon response (interferon)) [15]. For each immune module, metagene expressions were always significantly higher in C3 compared to C2 (Additional file 7). Based on a variety of immune cells and immune processes, these results demonstrated that high immune response (HIR) could be considered as a hallmark of C3. Considering C2 and C3 patients, the impact of Rody's metagenes on EFS and OS analyses was assessed. High expression of the majority of these metagenes was significantly associated with a better outcome: MHC-II (EFS: $P=0.0045$, hazard ratio $(\mathrm{HR})=0.61$; OS: $P=0.0117, \mathrm{HR}=$ 0.57 ), interferon (EFS: $P=0.007, \mathrm{HR}=0.66 ; \mathrm{OS}: P=$ $0.0138, \mathrm{HR}=0.63$ ), STAT1 (EFS: $P=0.004, \mathrm{HR}=0.68$; OS: $P=0.0069, \mathrm{HR}=0.64$ ), LCK (EFS: 0.0065, HR =0.66; OS: $P=0.0086, \mathrm{HR}=0.60)$ and HCK (EFS: $P=0.0276, \mathrm{HR}=$ 0.62; OS: $P=0.06$ ). A trend was found for MHC-I (EFS: $P=0.08$; OS: $P=0.14)$. IgG module was not associated with disease evolution (EFS: $P=0.69$; OS: $P=0.43$ ).

Ward's hierarchical clustering applied to these immune modules separated $\mathrm{C} 2$ and $\mathrm{C} 3$ patients into two clusters: one exclusively composed of $\mathrm{C} 3$ patients with HIR $(n=26)$ and the other composed of a majority (84\%) of C2 patients with low immune response (LIR) (Figure 4A). All TNBCtype immunomodulatory $(n=19)$ were included in the HIR group. EFS analysis showed that HIR patients had a significantly better outcome than LIR patients $(P=0.0025$; Figure $4 \mathrm{~B})$. Hence, immune response subtyping separated $\mathrm{TN}$ basal-like patients into two groups with different outcomes. A pooled cohort composed of $\mathrm{C} 2, \mathrm{C} 3$ and their equivalent in the external cohort, C2' and C3', $(n=141)$ confirmed this finding $(P=0.0212 ; n=70$ and 71 , respectively for LIR and HIR) (Additional file 8). In our study, Rody's metagenes were more powerful than Teschendordff's GES to separate $\mathrm{C} 2$ and $\mathrm{C} 3$ as a function of immune response.

M2/M1 GES Of the 649 genes found differentially expressed by the SAM method, 611 were measured in Affymetrix ${ }^{\circ}$ Human Genome U133 Plus 2.0 Arrays and used for macrophage subtyping (Additional file 9). Seventy-nine genes were in common between M2/M1 GES and the C2 versus $\mathrm{C} 3$ genes list, which included most differentially expressed genes between $\mathrm{C} 2$ and $\mathrm{C} 3$. Considering only $\mathrm{C} 2$ and $\mathrm{C} 3$ clusters, M2/M1 GES was significantly associated with a bad outcome $(P=0.02$ and 0.04 , respectively, for EFS and OS). M2 pro- 


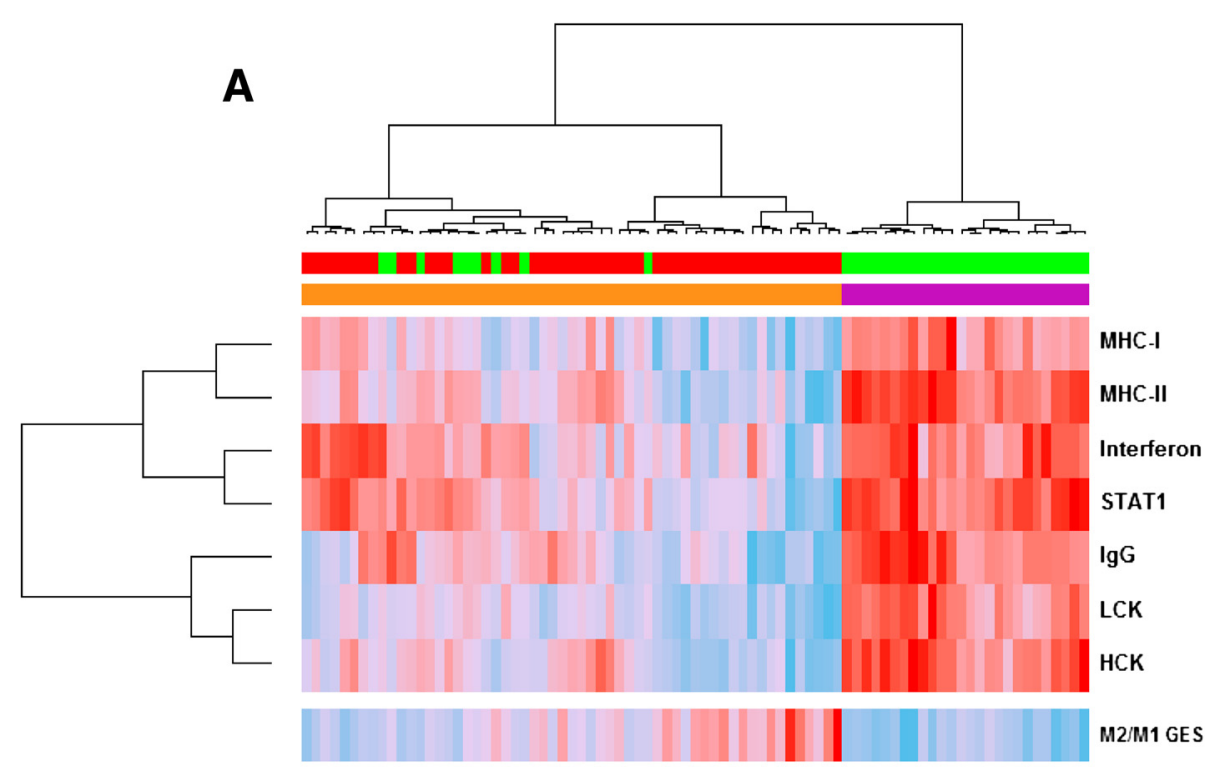

B

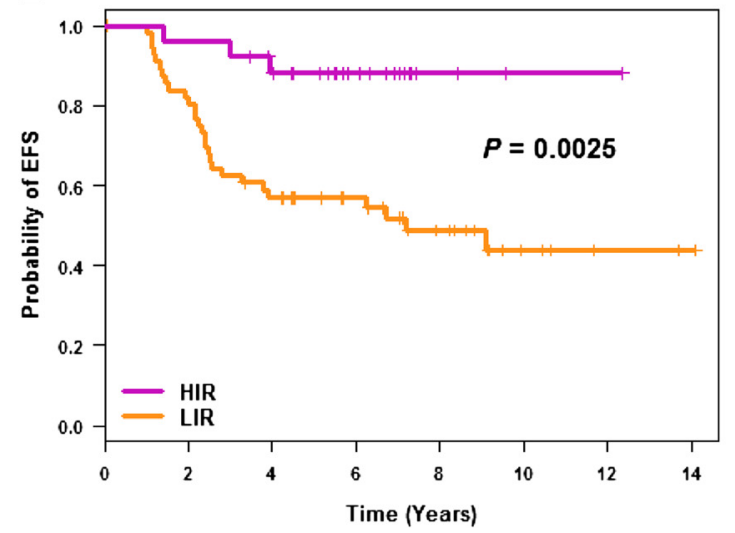

Figure 4 C2/C3 immune response dissection. (A) Ward's hierarchical clustering and heatmap showing the segregation of C2 (red) and C3 (green) patients as a function of the seven Rody's metagenes (B lymphocytes (lgG); macrophages and monocyte/myeloid lineage cells (HCK); professional antigen-presenting cells (MHC-II); T-cell (LCK); cell types for presentation of intracellular antigens (MHC-I); interferon signal transduction (STAT1); interferon response (interferon)) and M2/M1 gene-expression signature (GES) (continuous colour scale, from minimum (8.05, 6.90, 5.06, 7.84, 7.43, 9.68, 11.19 and -1.36 for HCK, LCK, IgG, STAT1, Interferon, MHC-II, MHC-I and M2/M1 GES, respectively; deep sky blue) to maximum (11.46, 12.24, 12.93, 13.21, 12.55, 13.75, 14.69 and -0.68 for HCK, LCK, IgG, STAT1, Interferon, MHC-II, MHC-I and M2/M1 GES, respectively; red). (B) Kaplan-Meier curves for event-free survival (EFS) analysis of breast cancer patients with high immune response (HIR) and low immune response (LIR).

tumourigenic macrophage genes were more expressed in C2 $(P<0.0001)$, which was characterized by LIR and bad outcome, and M1 tumour suppressor macrophage genes were more expressed in $\mathrm{C} 3$, which was characterized by HIR and better outcome. Of note, we did not find any correlation between VEGF score and M2 macrophage signature $(\mathrm{r}=0.08 ; P=0.45)$.

\section{Single gene-expression intuitive approach}

C1 was characterized by overexpression of luminal markers (AR, ESR1, GATA3, KRT18, KRT19 and MUC1) and low expression of basal-like markers (CDH3, KIT, KRT5, KRT6B, KRT14, KRT17) compared to C2 and C3, leading us to name it LAR (Additional file 10). ESR1 distribution according to the three clusters showed a significantly higher expression in $\mathrm{C} 1$ compared to $\mathrm{C} 2$ $(P=0.0022)$ and a trend with $\mathrm{C} 3(P=0.07) . E R B B 2$ was only found overexpressed between $\mathrm{C} 1$ and $\mathrm{C} 3$. Higher expression of "epithelial cell-cell adhesion" (CDH1 and $E P C A M)$ and proliferation markers (MKI67, UBE2C, $R A C G A P 1)$ was observed between $\mathrm{C} 2$ and $\mathrm{C} 3$. Low expression of EMT (CDH2, TGFB1), "immune system response" (CD4, CD79A, CXCL2, IL6, VAV1), breast stem cells $(A B C A 8, A L D H 1 A 1)$ and angiogenesis markers (TEK, TIE1, VEGFC) in $\mathrm{C} 2$ differentiated this cluster from $\mathrm{C} 3$. In regard of these results, we may conclude 
that $\mathrm{C} 2$ is a true "basal-like" cluster. As detailed above, biological behaviour of $\mathrm{C} 3$ was different from C2. Most of the underlined differences, except the three claudin genes (CDLN3, CDLN 4 and CDLN7), characterized a "claudin-low" cluster that expressed low luminal differentiation markers, high EMT markers, immune response genes, cancer stem cell-like features and a stem cellassociated biological process. In regard to a preponderance of immune response genes in $\mathrm{C} 3$, we thought that "immune-related" was more suited to this last cluster.

\section{Gene ontology biological process terms enrichment}

One overexpressed gene probe cluster was identified for each cluster of patients by means of the $5 \%$ most variant gene probes $(n=2,734)$ heatmap. Those clusters were named $\mathrm{H} 1$ for $\mathrm{C} 1, \mathrm{H} 2$ for $\mathrm{C} 2$ and $\mathrm{H} 3$ for $\mathrm{C} 3 . \mathrm{H} 1, \mathrm{H} 2$ and $\mathrm{H} 3$ were composed of 481, 442 and 222 gene probes, respectively (Additional file 11). Lists of discriminating gene probes $(\mathrm{C} 1$ versus $\mathrm{C} 2, \mathrm{C} 1$ versus $\mathrm{C} 3, \mathrm{C} 2$ versus $\mathrm{C} 3$ and $\mathrm{C} 1$ versus $\mathrm{C} 2$ and $\mathrm{C} 3$ ) were composed of 682, 575, 565 and 565 gene probes, respectively. Twentyone GO biological process terms enrichment analyses demonstrated that $\mathrm{C} 1$ was characterized by epithelial cell differentiation and a hormone metabolic process, $\mathrm{C} 2$ by cell adhesion, locomotion and chemotaxis, and C3 by immune response (Additional file 12).

\section{External validation}

Eighty-seven TN patients from GSE21653 were used for external validation. The same global process, as described for our cohort, was used. Results are summarized in Figure 5 and detailed in Additional file 13. In accordance with the clustering results of our cohort, GSE21653 TN patients were divided up into one nonbasal-like cluster $\left(\mathrm{Cl}^{\prime}\right)$ and two basal-like clusters (one named true basal-like or basal-like $\left(\mathrm{C}^{\prime}\right)$ and the other, which included claudin-low subtypes $(28 \%)$, characterized by immune response $\left(\mathrm{C}^{\prime}\right)$ ). One difference was that the GSE21653 cohort included PAM50 HER2-E subtypes in $\mathrm{C}^{\prime}$ '. Moreover, only five patients $(5.74 \%)$ were differently classified with PAM.

\section{Discussion}

Our work has once again raised the question of finding a gold standard method for breast cancer molecular subtyping; personalized medicine cannot do without effective identification of patients. We conducted an unsupervised analysis of microarray gene-expression profiles of a TN cohort composed of 107 patients. The robustness of our results was based on concordance of numerous and complementary functional annotation means: bio-clinical data (age, histological grade, NPI score), immunohistochemistry markers (CK5, HER1, Ki67, AR and FOXA1), 17 GES, intuitive single gene-expression approach and GO enrichment. Furthermore, our analysis was reinforced by external validation which confirmed our results. Functional annotation of clusters obtained by means of fuzzy clustering showed that $22 \%$ of these patients were not basal-like $(\mathrm{C} 1=\mathrm{LAR})$, which is in concordance with other works [30]. Of note, in GSE21653, 20 to 30\% of SSP classified C1' patients were subtyped as HER2-E; ERBB2 expression was in concordance with this result (Additional file 13). As a consequence of this, the $\mathrm{TN} \mathrm{C} 1$ cluster should be named "non-basal-like", because it might be composed of non-basal-like patients with LAR and/or HER2-E characteristics. The two other clusters were enriched in basal-like subtype: $\mathrm{C} 2$, which could be considered as an almost pure basal-like cluster, included patients with bad outcome, and C3, enriched in basal-like subtypes but to a lesser extent, included patients with better outcome. C3 was composed of $26 \%$ claudin-low subtypes. Of note, claudin-low patients were subtyped by means of GES. Our cohort was probably too small to individualize a well-separated claudin-low subtype; Prat and colleagues initially characterized claudin-low subtype in a cohort of 337 patients [6]. Dissection of immune response, which was high in these two last clusters compared to $\mathrm{C} 1$, was first performed by means of seven metagenes. Results showed that HIR was a hallmark of C3, and that HIR was associated with a better outcome than LIR (C2). This was true for our cohort $(P=0.0195)$ alone and pooled with GSE21653 TN patients $(P=0.002)$ (Figure 2, Additional file 4). We proposed that C3 should be called "HIR", because HIR had the clinical advantage of individualizing a cluster of patients with good outcome. C2 seemed to correspond to "basal-like immune-suppressed", and C3 to "basal-like immune-activated", recently described by Burstein and colleagues [31].

In addition to other studies, our work demonstrated that CK5 should be used rather than CK5/6 to identify basal-like subtype. Indeed, in our cohort, sensitivity (based on $\mathrm{C} 2$ and $\mathrm{C} 3$ results) was $83 \%$ for CK5 and only $34 \%$ for CK5/6; both markers had similar specificity (based on C1 results): $77 \%$ for CK5 and $82 \%$ for CK5/6. In C1, 5 cases out of 22 were positive for CK5 (23\%), 4/ 22 were positive for CK5/6 (18\%) and 11/22 were positive for HER1 (50\%). In 2004, Abd El-Rehim and colleagues showed that $27.4 \%$ of breast tumours displayed a combined luminal and basal phenotype (positivity for one or more of the luminal markers together with one or more of the basal markers) [32]. This could explain why CK5- or CK5/6-positive tumours were found in C1.

We focused on immune response, which is known to play a major role in tumour progression, because it was the main characteristic allowing us to distinguish between the two basal-like-enriched clusters: C2 (LIR) and C3 (HIR). Furthermore, actors or mechanisms of immune networks constitute potential drug targets suggesting that 


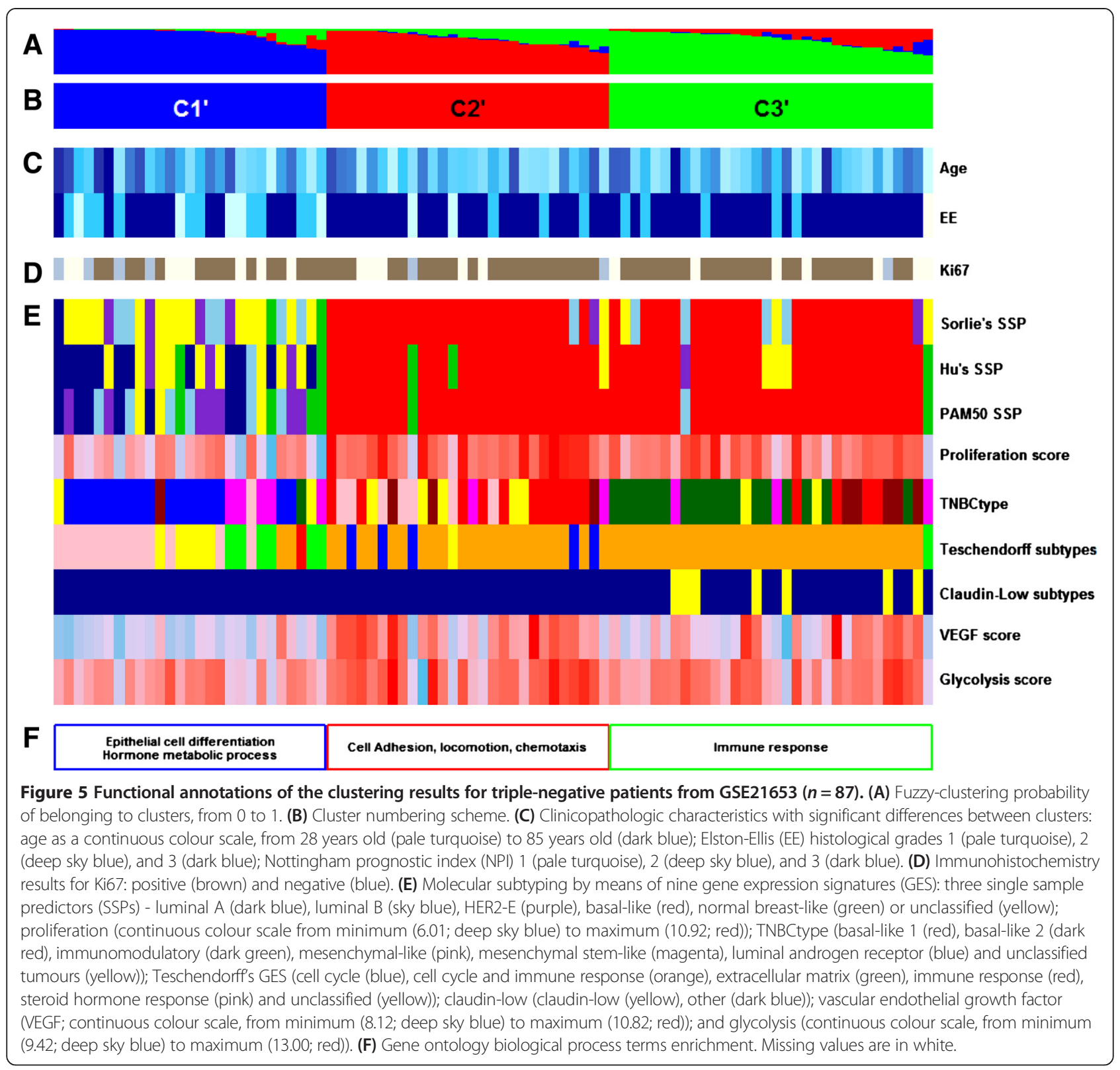

some TN patients might benefit from immune-based therapies. In cancer, some immune cells are known to induce anti-tumoural effects (natural killer cells, CD8+ T cells, Th1 cells, dendritic cells 1, M1 macrophages, and so forth), and others induce pro-tumoural effects (myeloidderived suppressor cells, CD4+ T cells, Th2 cells, dendritic cells 2, M2 macrophages, and so forth) [33,34]. These effects result from complex cross talk between immune cells, tumour cells and other cell populations of the microenvironment by means of extracellular signals, including many cytokines and their soluble receptors $[35,36]$. In breast cancer, recent works confirmed that tumour infiltrating lymphocytes (TILs) were most frequently found in highly proliferative tumours, including $\mathrm{TN}$, and were associated with a favourable outcome [37,38]. Today, TILs are becoming a new breast cancer marker, particularly in TN and HER2-positive breast cancer [33]. As recommended by an international working group of experts in 2014, TIL score must take into account all mononuclear cells including lymphocytes and plasma cells, and excludes granulocytes, other polymorphonuclear leukocytes, dendritic cells and macrophages [33]. Nevertheless, macrophages are key modulator and effector cells in immune response and tumour progression [35]. An old, but still used, classification of macrophages roughly separates them into two "extreme" polarized subpopulations: the classically activated type 1 macrophages (M1) and the alternative activated type 2 macrophages (M2). In 2010, Qian and 
Pollard described six macrophage functions (activated, immunosuppressive, angiogenic, metastasis-associated, perivascular and invasive) ascribed to a unique macrophage subpopulation [39]. Recently, a working group of macrophage biologists defined a nomenclature and experimental guidelines to take into account the heterogeneity of macrophage activation and polarization [40]. They recommended a nomenclature linked to the activation standards and counted seven human macrophage subpopulations, three M1 types and four M2 types [40]. Beside the extreme phenotypes, intermediate phenotypes of macrophages should also be evaluated when dissecting immune response in cancer [41,42]. Within this cell group, tumour-associated macrophages (TAMs), which are considered as M2-like, represent a major source of proteases involved in tumour progression [43]. Very synthetically, polarized macrophages, together with fibroblasts and vascular endothelial cells from the tumour microenvironment, in conjunction with tumour cells, intervene at different stages of the cancer process. They have been implicated in the angiogenic switch (high production of VEGF), local invasion, and metastasis. Thus it is important to take into account these cells when dissecting immune response, and to identify their subpopulations because they have negative and positive effects on cancer evolution.

In this work, HIR, objectivised by means of Rody's and TNBCtype GES, was exclusively related to "antitumoural" response; none of these GES were related to "pro-tumourigenic" response. Furthermore, Teschnedorff's GES was unable to dissect immune response; immune response was high in C2 and C3 (Figure 3E). Almost all Rody's immune modules showed that high expression was linked to good outcome (Figure 4). Expression of LCK and IgG Rody's metagenes, as markers for T and B cells, were in concordance with current knowledge. On the contrary, high expression of HCK, which take into account macrophages and monocyte/myeloid lineage cells, was associated with favourable outcome. This result was a bit surprising because, in breast cancer, TAMs are known to be associated with bad prognosis [34]. Our hypothesis was that HCK was unable to distinguish between the two extreme macrophage phenotypes. For this reason, we established a M2/M1 GES to subtype TAMs into M1-like or M2-like groups. According to new nomenclature, M2/M1 GES, which should be named M(IL-4)/M(IFN- $\gamma)$ GES, showed that M(IL-4) (M2 pro-tumourigenic macrophages) were more frequent in LIR (C2), which was characterized by a bad outcome, and that M(IFN- $\gamma$ ) (M1 tumour suppressor macrophages) were more frequent in HIR (C3), which was characterized by a favourable outcome. In other words, $\mathrm{C} 2$ was characterized by high protumourigenic and low anti-tumoural immune response and $\mathrm{C} 3$ by low pro-tumourigenic and high anti-tumoural immune response. Finally, these results are in concordance with current knowledge, and are of particular importance because M2 inhibition, which may play a major pro-tumourigenic role in $\mathrm{C} 2$, represents a potential therapeutic strategy. Repolarization of M2 into M1 macrophages and inhibition of M2 macrophages both represent promising ways to treat cancer $[44,45]$. Cancer treatment may take advantage of therapies that interfere with M2 macrophages. Although the following model was not based on breast cancer, but on nude mice xenografted with colon cancer cells, GTP cyclohydrolase (CGH1) inhibition by 2,4-diamino-6-hydroxypyrimidine reduced tumour growth and angiogenesis by shifting the phenotype of tumour-associated macrophages from proangiogenic M2 towards M1 [46].

However, some caution must be taken with our results. First, our main hypothesis was that immune cells and particularly macrophage subpopulations represented main key effectors of TN breast cancer. Today, numerous concordant studies support this hypothesis [47-51]. Second, we showed that the M2 macrophage geneexpression profile was associated with bad outcome, and the reverse for M1. At this step, we did not demonstrate a causal link between M2 macrophage cells and outcome. Further analyses are required to dissect M2dependent causal chains or systems that might explain breast cancer progression and evolution. Phenotypic markers should also be tested to validate macrophage distribution. Third, new TN patients are needed to test the strength of this partition.

\section{Conclusions}

We identified three subtypes of TN patients: 1) nonbasal-like (22\%); 2) basal-like with LIR and high M2-like macrophages (45\%); and 3) basal-enriched, including claudin-low subtypes, with HIR and low M2-like macrophages (33\%). Our study added another well-defined TN cohort to the scientific community. We showed that our gene-expression data could be pooled with others to strengthen unsupervised analyses and to feed databases [52]. As others have done, we concluded that around $25 \%$ of TN patients, including non-basal-like cluster (LAR in our study), could receive hormonotherapy or anti-HER2 therapy. Furthermore, based on concordance between robust molecular results and IHC, we showed that the CK5 antibody was better suited than the CK5/6 antibody to subtype basal-like patients. This result is of importance within the framework of the search for a TN subtyping gold standard method. In this work, we pointed out that macrophages, particularly M2-like, offered a variety of therapeutic targets in $\mathrm{TN}$ patients. In breast cancer, and particularly in cases of TN basal-like tumours, future clinical trials evaluating novel immune therapies will have to be set up with patients stratified 
by means of the composition of their leukocyte infiltrate, which should include macrophage subpopulation identification [53]. A lot of work needs to be done in order to define this new multiparametric biomarker panel. To this end, cytology together with expression microarray data deconvolution seem more likely to succeed $[54,55]$. To conclude, we hypothesize that TN basal-like treatment, among other things, will have to restore the normal balance of immune cells, by means of targeted immune cell inhibition and/or augmentation, and that immune therapy will become an important component of TN basal-like combined therapies.

\section{Additional files}

\section{Additional file 1: Details of antibodies used for immunohistochemistry. Additional file 2: Interpretation of immunohistochemical staining. Additional file 3: Gene-expression signatures (GES) used for fuzzy cluster functional annotation.}

Additional file 4: Kaplan-Meier curves for event-free survival analysis based on fuzzy-clustering partition. (A) GSE21653 triple negative (TN) breast cancer patients ( $n=87$ ). (B) Kaplan-Meier analysis of pooled cohorts (ours and GSE21653; $n=194$ ) shows significantly increased event-free survival in $C 3$ patients compared to $C 1(P=0.03)$ and $C 2$ patients $(P=0.002)$.

Additional file 5: Distribution of single sample predictor (SSP) subtypes according to the three clusters of our cohort. (A) Sorlie's SSP. (B) Hu's SSP. (C) Parker's SSP (PAM50). Bar graph plots shows the distribution of intrinsic subtypes: luminal A (dark blue) and B (light blue), HER2-E (purple), basal-like (red), normal breast-like (green) or unclassified (yellow) tumours within each cluster. Whatever the SSP, subtype distributions show that C1 is a luminal-enriched cluster and not a basal-like cluster, C2 is an almost pure basal-like cluster, and C3 is a basal-like-enriched cluster.

Additional file 6: Distribution of TNBCtype subtypes according to the three clusters of our cohort. Bar graph plots show the distribution of the six TNBCtype subtypes: basal-like 1 (red), basal-like 2 (dark red), immunomodulatory (dark green), mesenchymal-like (pink), mesenchymal stem-like (magenta), luminal androgen receptor (blue) and unclassified tumours (yellow), within each cluster.

Additional file 7: Rody's metagene expression levels according to our cohort's clusters. For each immune module, $P$ value of Tukey's test for the comparison between C2 and C 3 is indicated. Metagene expressions are always significantly higher in C3 compared to C2.

Additional file 8: Kaplan-Meier curves for event-free survival analysis based on Rody's metagenes clustering. Rody's metagenes clustering was based on patients included in C2 and C3 (our cohort; see Figure 4A), and in C2' and C3' (GSE21653) (low immune response (LIR), $n=70$; high immune response $(H \mathrm{R}), n=71)$.

Additional file 9: M2/M1 gene expression signature (GES) genes list. List of the 611 genes used to compute the M2/M1 GES and their relative expression in $\mathrm{M} 2$ versus $\mathrm{M} 1$ population.

Additional file 10: Expression level of chosen genes between the three fuzzy clusters of our cohort.

Additional file 11: Fuzzy clustering heatmap of the 5\% most variant gene probes. Each column represents a patient $(n=107)$ and each row an Affymetrix gene probe $(n=2,734)$. Green indicates low expression, red indicates high expression. Clusters of overexpressed genes used for gene ontology (GO) biological process terms enrichment are indicated by a bracket: $\mathrm{H}_{1}$ for $\mathrm{C} 1$ (blue), $\mathrm{H}_{2}$ for $\mathrm{C} 2$ (red) and $\mathrm{H} 3$ for $\mathrm{C} 3$ (green). $\mathrm{H} 1, \mathrm{H} 2$ and $\mathrm{H} 3$ were composed of 481, 442 and 222 gene probes, respectively.

Additional file 12: Biological process gene ontology (GO) terms enrichment for our cohort.
Additional file 13: External validation process results. Eighty-seven triple negative (TN) patients of GSE21653 cohort were used for external validation. The same global process, as described for our cohort, was used and is described thereafter.

\section{Abbreviations}

AR: androgen receptor; EFS: event-free survival; EMT: epithelial-to-mesenchymal transition; ER: oestrogen receptor; GEO: Gene Expression Omnibus; GEP: gene-expression profiling; GES: gene-expression signatures; GO: gene ontology; IHC: immunohistochemistry; HER2-E: HER2-enriched; HES: hemaluneosin-safran; HIR: high immune response; HR: hazard ratio; LAR: luminal androgen receptor; LIR: low immune response; NPI: Nottingham prognostic index; OS: overall survival; PAM: prediction analysis for microarrays;

PR: progesterone receptor; SAM: significance analysis of microarrays; SBR: Scarff Bloom Richardson; SSP: single sample predictor; TAM: tumour-associated macrophage; TIL: tumour infiltrating lymphocyte; TN: triple-negative; TNBCtype: a subtyping tool for triple-negative breast cancer; VEGF: vascular endothelial growth factor.

\section{Competing interests}

The authors declare that they have no competing interests.

\section{Authors' contributions}

PJ and MC conceived and designed the study. CGC, LC, AV and HL carried out statistical analyses. WG performed bioinformatics. DL, IV and W carried out immunohistochemistry. PJ, LC, WG and CG provided patients and obtained bio-clinical data. All authors have been involved in drafting the manuscript. All authors read and approved the final manuscript.

\section{Acknowledgements}

We wish to thank Pr Charles Perou and Dr Aleix Prat for their valuable help. Tissues used in this work were provided by: Institut Régional du Cancer Nantes-Atlantique tumour bank, funded by the Institut National du Cancer (INCa) and the Cancéropôle Grand Ouest, and Anjou Maine ICO Paul Papin tumour bank funded by the INCa. We thank the transcriptomic team (Elise Douillard, Magali Devic, Emilie Moranton and Nathalie Roi), the immunohistochemistry team (Nadège Friquet), the IRCNA tumour bank team (Estelle Biraud and Vanessa Veron,) and the Anjou Maine ICO Paul Papin tumour bank team (Dr Agnès Chassevent) for technical assistance.

\section{Author details}

${ }^{1}$ Unité Mixte de Génomique du Cancer, Institut de Cancérologie de l'Ouest site René Gauducheau, Bd J. Monod, 44805 Saint Herblain Cedex, France. ${ }^{2}$ Unité de Bioinfomique, Institut de Cancérologie de l'Ouest - site René Gauducheau, Bd J. Monod, 44805 Saint Herblain Cedex, France. ${ }^{3}$ Département de Biopathologie, Institut de Cancérologie de l'Ouest - site René Gauducheau, Bd J. Monod, 44805 Saint Herblain Cedex, France. ${ }^{4}$ INSERM U892, IRT-UN, 8 quai Moncousu, 44007 Nantes Cedex, France. ${ }^{5}$ Service d'Anatomie Pathologique B, Hôpital G\&R Laënnec, Bd J. Monod, 44805 Saint Herblain, France. ${ }^{6}$ Unité de Biostatistique, Institut de Cancérologie de l'Ouest - site René Gauducheau, Bd J. Monod, 44805 Saint Herblain Cedex, France. ${ }^{7}$ Sorbonne Universités, UPMC University, Département de Biostatistique, 4 place Jussieu, 75005 Paris, France. ${ }^{8}$ AP-HP, University Hospitals Pitié-Salpêtrière Charles-Foix, Département de Biostatistique, Santé Publique et Informatique Médicale, 47-83 Bd de l'Hôpital, 75013 Paris, France. ${ }^{9}$ Institut de Cancérologie de l'Ouest - site Paul Papin, 2 rue Moll, 49933 Angers Cedex 9, France. ${ }^{10}$ Laboratoire d'Anatomie Pathologique, Institut de Cancérologie de l'Ouest - site Paul Papin, 2 rue Moll, 49933 Angers Cedex 9, France. ${ }^{11}$ Service d'Oncologie Médicale, Institut de Cancérologie de I'Ouest - site René Gauducheau, Bd J. Monod, 44805 Saint Herblain Cedex, France.

Received: 4 November 2014 Accepted: 10 March 2015 Published online: 20 March 2015

\section{References}

1. Foulkes WD, Smith IE, Reis-Filho JS. Triple-negative breast cancer. N Engl J Med. 2010;363:1938-48.

2. Perou CM, Sorlie T, Eisen MB, van de Rijn M, Jeffrey SS, Rees CA, et al. Molecular portraits of human breast tumours. Nature. 2000;406:747-52 
3. Sorlie T, Perou CM, Tibshirani R, Aas T, Geisler S, Johnsen H, et al. Gene expression patterns of breast carcinomas distinguish tumor subclasses with clinical implications. Proc Natl Acad Sci U S A. 2001;98:10869-74.

4. Prat A, Perou CM. Deconstructing the molecular portraits of breast cancer. Mol Oncol. 2011;5:5-23.

5. Herschkowitz JI, Simin K, Weigman VJ, Mikaelian I, Usary J, Hu Z, et al. Identification of conserved gene expression features between murine mammary carcinoma models and human breast tumors. Genome Biol. 2007;8:R76.

6. Prat A, Parker JS, Karginova O, Fan C, Livasy C, Herschkowitz Jl, et al. Phenotypic and molecular characterization of the claudin-low intrinsic subtype of breast cancer. Breast Cancer Res. 2010;12:R68.

7. Hu Z, Fan C, Oh DS, Marron JS, He X, Qaqish BF, et al. The molecular portraits of breast tumors are conserved across microarray platform. BMC Genomics. 2006;7:96.

8. Chen X, Li J, Gray WH, Lehmann BD, Bauer JA, Shyr Y, et al. TNBCtype: a subtyping tool for triple-negative breast cancer. Cancer Inform. 2012;11:147-56.

9. Sabatier R, Finetti P, Cervera N, Lambaudie E, Esterni B, Mamessier E, et al. A gene expression signature identifies two prognostic subgroups of basal breast cancer. Breast Cancer Res Treat. 2011;126:407-20.

10. Sabatier R, Finetti P, Adelaide J, Guille A, Borg JP, Chaffanet M, et al. Downregulation of ECRG4, a candidate tumor suppressor gene, in human breast cancer. PLoS One. 2011:6:e27656.

11. Sorlie T, Tibshirani R, Parker J, Hasties T, Marron JS, Nobel A, et al. Repeated observation of breast tumor subtypes in independent gene expression data sets. Proc Natl Acad Sci U S A. 2003;100:8418-23.

12. Teschendorff AE, Miremadi A, Pinder SE, Ellis IO, Caldas C. An immune response module identifies a good prognosis subtype in estrogen negative breast cancer. Genome Biol. 2007;8:R157.

13. Hu Z, Fan C, Livasy C, He X, Oh DS, Ewend MG, et al. A compact VEGF signature associated with distant metastases and poor outcomes. BMC Med. 2009;7:9.

14. Parker JS, Mullins M, Cheang MC, Leung S, Voduc D, Vickery T, et al. Supervised risk predictor of breast cancer based on intrinsic subtypes. J Clin Oncol. 2009;27:1160-7.

15. Rody A, Holtrich U, Pusztai L, Liedtke C, Gaetje R, Ruckhaeberle E, et al. T-cel metagene predicts a favorable prognosis in estrogen receptor-negative and HER2-positive breast cancers. Breast Cancer Res. 2009;11:R15.

16. Dexter TJ, Sims D, Mitsopoulos C, Mackay A, Grigoriadis A, Ahmad AS, et al. Genomic distance entrained clustering and regression modelling highlights interacting genomic regions contributing to proliferation in breast cancer. BMC Syst Biol. 2010;4:127

17. Beyer M, Mallmann MR, Xue J, Staratschek-Jox A, Vorholt D, Krebs W, et al. Highresolution transcriptome of human macrophages. PLoS One. 2012;7:e45466.

18. Lehmann BD, Bauer JA, Chen X, Sanders ME, Chakravarthy AB, Shyr Y, et al. Identification of human triple-negative breast cancer subtypes and preclinical models for selection of targeted therapies. J Clin Invest. 2011;121:2750-60.

19. Huang DW, Sherman BT, Lempicki RA. Systematic and integrative analysis of large gene lists using DAVID bioinformatics resources. Nat Protoc. 2009;4:44-57.

20. Huang DW, Sherman BT, Lempicki RA. Bioinformatics enrichment tools: paths toward the comprehensive functional analysis of large gene lists. Nucleic Acids Res. 2009:37:1-13.

21. Eden E, Navon R, Steinfeld I, Lipson D, Yakhini Z. Gorilla: a tool for discovery and visualization of enriched $\mathrm{GO}$ terms in ranked gene lists. BMC Bioinformatics. 2009;10:48.

22. Chen J, Bardes EE, Aronow BJ, Jegga AG. ToppGene Suite for gene list enrichment analysis and candidate gene prioritization. Nucleic Acids Res. 2009;37:W305-11.

23. R Core Team. R: a language and environment for statistical computing. $\mathrm{R}$ Foundation for Statistical Computing, Vienna, Austria; 2014. http://www.Rproject.org/.

24. Nielsen TO, Hsu FD, Jensen K, Cheang M, Karaca G, Hu Z, et al. Immunohistochemical and clinical characterization of the basal-like subtype of invasive breast carcinoma. Clin Cancer Res. 2004;10:5367-74.

25. Bhargava R, Beriwal S, McManus K, Dabbs DJ. CK5 is more sensitive than CK5/6 in identifying the "basal-like" phenotype of breast carcinoma. Am J Clin Pathol. 2008;130:724-30.

26. Alshareeda AT, Soria D, Garibaldi JM, Rakha E, Nolan C, Ellis IO, et al. Characteristics of basal cytokeratin expression in breast cancer. Breast Cancer Res Treat. 2013;139:23-37.
27. Weigelt B, Mackay A, A'hern R, Natrajan R, Tan DS, Dowsett M, et al. Breast cancer molecular profiling with single sample predictors: a retrospective analysis. Lancet Oncol. 2010;11:339-49.

28. Ghabach B, Anderson WF, Curtis RE, Huycke MH, Lavigne JA, Dores GM. Adenoid cystic carcinoma of the breast in the United States (1977 to 2006): a population-based cohort study. Breast Cancer Res. 2010;12:R54.

29. Masuda H, Baggerly KA, Wang Y, Zhang Y, Gonzalez-Angulo AM, Meric-Bernstam $F$, et al. Differential response to neoadjuvant chemotherapy among 7 triplenegative breast cancer molecular subtypes. Clin Cancer Res. 2013;19:5533-40.

30. Prat A, Adamo B, Cheang MC, Anders CK, Carey LA, Perou CM. Molecular characterization of basal-like and non-basal-like triple-negative breast cancer. Oncologist. 2013;18:123-33.

31. Burstein MD, Tsimelzon A, Poage GM, Covington KR, Contreras A, Fuqua S, et al. Comprehensive genomic analysis identifies novel subtypes and targets of triple-negative breast cancer. Clin Cancer Res. 2015 21:OF1-OF11.

32. Abd El-Rehim DM, Pinder SE, Paish CE, Bell J, Blamey RW, Robertson JF, et al. Expression of luminal and basal cytokeratins in human breast carcinoma. J Pathol. 2004:203:661-71.

33. Salgado R, Denkert C, Demaria S, Sirtaine N, Klauschen F, Pruneri G, et al Harmonization of the evaluation of tumor infiltrating (TILs) in breast cancer: recommendations by an international TILs-working group 2014. Ann Oncol. 2014;26:259-71.

34. Finak G, Bertos N, Pepin F, Sadekova S, Souleimanova M, Zhao H, et al. Stromal gene expression predicts clinical outcome in breast cancer. Nat Med. 2008;14:518-27.

35. Quail DF, Joyce JA. Microenvironmental regulation of tumor progression and metastasis. Nat Med. 2013;19:1423-37.

36. Obeid E, Nanda R, Fu YX, Olopade OI. The role of tumor-associated macrophages in breast cancer progression (review). Int J Oncol. 2013:43:5-12.

37. Loi S, Michiels S, Salgado R, Sirtaine N, Jose V, Fumagalli D, et al. Tumor infiltrating lymphocytes are prognostic in triple negative breast cancer and predictive for trastuzumab benefit in early breast cancer: results from the FinHER trial. Ann Oncol. 2014;8:1544-50.

38. Adams S, Gray RJ, Demaria S, Goldstein L, Perez EA, Shulman LN, et al. Prognostic value of tumor-infiltrating lymphocytes in triple-negative breast cancers from two phase III randomized adjuvant breast cancer trials: ECOG 2197 and ECOG 1199. J Clin Oncol. 2014;55:0491.

39. Qian BZ, Pollard JW. Macrophage diversity enhances tumor progression and metastasis. Cell. 2010;141:39-51.

40. Murray PJ, Allen JE, Biswas SK, Fisher EA, Gilroy DW, Goerdt S, et al. Macrophage activation and polarization: nomenclature and experimental guidelines. Immunity. 2014;41:14-20.

41. Gordon S, Martinez F. Alternative activation of macrophages: mechanism and functions. Immunity. 2010;32:593-604

42. Lawrence T, Natoli G. Transcriptional regulation of macrophage polarization: enabling diversity with identity. Nat Rev Immunol. 2011;11:750-61

43. Gocheva V, Wang HW, Gadea BB, Shree T, Hunter KE, Garfall AL, et al. IL-4 induces cathepsin protease activity in tumor-associated macrophages to promote cancer growth and invasion. Genes Dev. 2010;24:241-55.

44. Heusinkveld M, van der Burg SH. Identification and manipulation of tumo associated macrophages in human cancers. J Transl Med. 2011:9:216.

45. Luo Y, Zhou H, Krueger J, Kaplan C, Lee SH, Dolman C, et al. Targeting tumor-associated macrophages as a novel strategy against breast cancer. J Clin Invest. 2006;116:2132-41.

46. Pickert G, Lim HY, Weigert A, Häussler A, Myrczek T, Waldner M, et al. Inhibition of GTP cyclohydrolase attenuates tumor growth by reducing angiogenesis of tumor associated macrophages. Int J Cancer. 2013;132:591-604.

47. Beck AH, Espinosa I, Edris B, Li R, Montgomery K, Zhu S, et al. The macrophage colony-stimulating factor 1 response signature in breast carcinoma. Clin Cancer Res. 2009:15:778-87.

48. Campbell MJ, Tonlaar NY, Garwood ER, Huo D, Moore DH, Khramtsov Al, et al. Proliferating macrophages associated with high grade, hormone receptor negative breast cancer and poor clinical outcome. Breast Cancer Res Treat. 2011;128:703-11.

49. Medrek C, Ponten F, Jirström K, Leandersson K. The presence of tumor associated macrophages in tumor stroma as a prognostic marker for breast cancer patients. BMC Cancer. 2012;12:306.

50. Chaturvedi P, Gilkes DM, Takano N, Semenza GL. Hypoxia-inducible factordependent signaling between triple-negative breast cancer cells and mesenchymal stem cells promotes macrophages recruitment. Proc Natl Acad Sci U S A. 2014:11:E2120-9. 
51. Karn T, Pusztai L, Holtrich U, Iwamoto T, Shiang CY, Schmidt M, et al. Homogeneous datasets of triple-negative breast cancers enable the identification of novel prognostic and predictive signatures. PLoS One. 2011;6:e28403.

52. Jézéquel P, Campone M, Gouraud W, Guérin-Charbonnel C, Leux C, Ricolleau G, et al. bc-GenExMiner: an easy-to-use online platform for gene prognostic analyses in breast cancer. Breast Cancer Res Treat. 2012:131:765-75

53. André F, Dieci MV, Dubsky P, Sotiriou C, Curigliano G, Denkert C, et al. Molecular pathways: involvement of immune pathways in the therapeutic response and outcome in breast cancer. Clin Cancer Res. 2013;19:28-33.

54. Abbas AR, Wolslegel K, Seshasayee D, Modrusan Z, Clark HF. Deconvolution of blood microarray data identifies cellular activation patterns in systemic lupus erythematosus. PLoS One. 2009;: :e6098.

55. Shannon CP, Balshaw R, Ng RT, Wilson-McManus JE, Keown P, McMaster R, et al. Two-stage, in silico deconvolution of the lymphocyte compartment of the peripheral whole blood transcriptome in the context of acute kidney allograft rejection. PLoS One. 2014;9:e95224.

\section{Submit your next manuscript to BioMed Central and take full advantage of:}

- Convenient online submission

- Thorough peer review

- No space constraints or color figure charges

- Immediate publication on acceptance

- Inclusion in PubMed, CAS, Scopus and Google Scholar

- Research which is freely available for redistribution 